\title{
Exploration of training needs of school nursing teams in Leicester, Leicestershire and Rutland for development of training resources to support their role in tackling childhood obesity and weight management
}

\author{
Diane Talbot and Nikki Hill \\ Leicestershire Nutrition and Dietetic Service, Leicester, UK
}

With one-third of British children currently overweight or obese and prospected doubling of this number by 2050, school nurses (SN) will be expected to play an integral role in tackling childhood obesity. Healthy Weight, Healthy Lives: A Cross-Government Strategy for England outlines immediate plans for SN teams to 'give better information about children's health by providing parents with their child's results from the National Child Measurement Programme accompanied by help and signposting to support parents in addressing concerns about their child's weight ${ }^{\text {(1) }}$.

Commissioned by the Government Office East Midlands Public Health Team, a training resource is in development to support SN teams in taking an evidence-based practical approach to tackling childhood obesity. A needs assessment, by form of questionnaire, was carried out to ensure appropriateness of the training resource.

A questionnaire assessing current practice, perceived confidence in practice and training and resource needs in dealing with childhood obesity was distributed to all SN and SN assistants (SNA) in Leicestershire and Rutland ( $n$ 104). Responses were anonymous. Completed questionnaire results were inputted and analysed using SPSS statistical software (SPSS Inc., Chicago, IL, USA).

Of the questionnaires $75 \%$ were completed and returned. SN and SNA in Leicester, Leicestershire and Rutland at present provide services to an average of three schools with $81 \%$ currently delivering advice on healthy eating. Presently, less than half provide weight management advice for children. While $80 \%$ felt confident in providing advice on children's diets, one-third described themselves as not confident in advising on individual weight issues. Of the SN and SNA 36\% did not feel confident in raising the issue of overweight and obesity with children, this percentage increased to $>50$ when involving parents. Of the SN and SNA $>80 \%$ identified their training needs to include current healthy eating recommendations for children, weight management for children, behaviour-change techniques, parenting skills and current physical activity guidelines. The background and context of childhood obesity would be an important part of the training resource for $76 \%$ of the responders. Feedback suggests the need for training to include signposting to free reproducible resources including leaflets, games and web-based resources.

The emerging themes from the needs-assessment questionnaires suggest the need for a comprehensive SN team training package to encompass current evidence and guidelines relating to childhood obesity and dietary change. As SN teams become increasingly involved in front-line prevention and management of childhood obesity, the training package must provide practical skills and advice to increase confidence to raise the issue of overweight and obesity with children and parents while ensuring that the teams have an appropriate pool of free and easily-accessible resources to support them. A pilot of the training is to be delivered to SN teams in Leicestershire and Rutland by Leicestershire Nutrition and Dietetic Service in spring 2008.

1. Department of Health (2008) Healthy weight, healthy lives: a cross government strategy for England. http://www.dh.gov.uk/en/Publicationsandstatistics/ Publications/PublicationsPolicyAndGuidance/DH_082378 (accessed March 2008). 